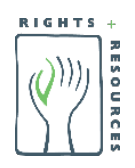

INITIATIVE DES DROITS ET RESSOURCES | OCTOBRE 2020

\title{
Crise de la COVID-19 sur fond de lois et règlementations préjudiciables en Asie du Sud et du Sud-Est
}

Asia Indigenous Peoples Pact (Pacte asiatique des peuples autochtones)

Coalition sur la tenure en Indonésie

Initiative des Droits et Ressources 


\section{Sommaire}

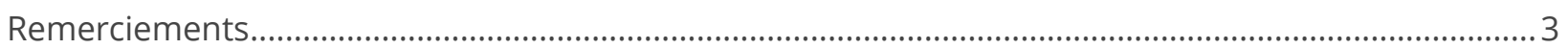

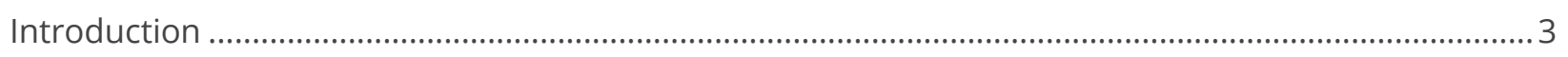

Droits humains pendant la COVID-19 : fermeture des espaces démocratiques.......................................5

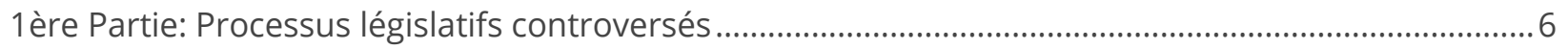

2ème Partie: Relance et compensation des entreprises ................................................................... 8

3ème Partie: Face à la pandémie, des "solutions" qui sapent les droits des PA / CL ............................11

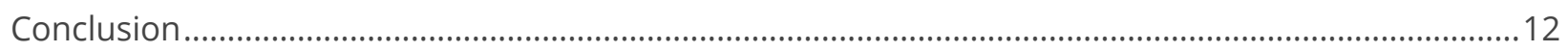




\section{Remerciements}

Les auteurs tiennent à remercier les avocats qui ont généreusement donné de leur temps et de leur perspicacité pour ce rapport : Asep Y Firdaus, expert juridique et directeur de l'Institut Epistema ; Andiko, SH. MH, juriste indonésien spécialiste du développement durable ; Shomona Khanna, avocate, Cour suprême de I'Inde ; Antonio G. M. La Viña et Joy Reyes, Observatoire de Manille.

Nous remercions également les traducteurs qui ont contribué à mettre ce rapport à la disposition des locuteurs de philippin, de bahasa Indonesia, d'hindi, de français et d'espagnol : Jo Ann Guillao ; Septiari Ayu ; Hridayesh Joshi ; Dhruva Narayan ; Samuel Tribollet ; et Pauline Kulstad.

\section{Introduction}

La Planète est en proie à une crise sanitaire mondiale sans précédent suite à la propagation de la COVID-19. A la date du 29 septembre, plus d'un million de personnes sont mortes à cause du virus. Une pandémie prolongée est de plus en plus probable, car la crise sanitaire initiale est en train de se transformer en une combinaison de crise de sécurité alimentaire et de crise économique.

Parmi les groupes les plus vulnérables à la pandémie de la COVID-19 figurent les Peuples Autochtones et les Communautés Locales (PA et $\mathrm{CL}$ ), des groupes qui sont déjà victimes d'inégalités, de stigmatisation et de discrimination. Entre autres problèmes structurels qui entravent les capacités de ces communautés à faire face à la pandémie, on peut citer le manque d'accès aux services de santé de base, aux installations sanitaires et à Internet pour utiliser les plateformes éducatives en ligne. Cependant, lorsque les communautés jouissent d'un droit à l'autodétermination, elles ont montré toute leur ténacité en proposant des réponses culturellement appropriées et pilotées par la communauté, même face à la COVID-19.

Une réponse efficace à cette crise passera fondamentalement par des droits fonciers garantis, des écosystèmes sains et productifs, et le respect de la volonté d'une communauté de rester volontairement isolée en arrêtant tous les projets nécessitant son consentement libre informé et préalable (CLIP) pendant toute la durée de la pandémie.

Les PA et les CL gèrent de façon coutumière plus de $50 \%$ de la surface terrestre du globe, mais seulement $10 \%$ de cette surface sont sous un régime de tenure qui garantit leurs droits de propriété traditionnels. ${ }^{1}$ Cette tenure précaire limite le pouvoir des PA et des CL de mettre en œuvre des stratégies bien établies aptes à empêcher la propagation d'agents pathogènes virulents, comme par exemple l'isolement volontaire. ${ }^{2}$ En outre, en l'absence d'une tenure stable, les territoires des communautés sont menacés à cause de leurs nombreuses ressources naturelles, car dans le souci de

\footnotetext{
${ }^{1}$ La reconnaissance de droits collectifs garantis sur les terres et les ressources est largement perçue par les acteurs étatiques et non étatiques comme contribuant à l'avancement des objectifs sociaux, économiques et environnementaux définis au niveau international. Dans la pratique, les organes législatifs nationaux ont été lents à formaliser les régimes de tenure qui soutiennent les communautés et les pratiques traditionnelles des Peuples Autochtones, des Communautés Locales et des femmes en milieu rural.

${ }^{2}$ Les villages tribaux du Bengale occidental en Inde ont été proactifs face à la COVID-19 en imposant leurs propres confinements et en isolant les travailleurs migrants revenant des villes.
} 
juguler la récession économique mondiale, les gouvernements mettent l'accent sur le développement de l'industrie extractive. Enfin, la pandémie a entraîné une baisse de l'application des lois sur les droits fonciers, une hausse de l'accaparement des terres et la criminalisation des PA et des CL luttant pour leurs droits fondamentaux sur les systèmes socio-écologiques qu'ils gèrent. ${ }^{3}$ Ainsi, au-delà de ses répercussions sur la santé publique, la COVID-19 est un "multiplicateur de menaces" sur les terres sans tenure garantie.

La récente publication du cinquième rapport sur les Perspectives mondiales de la diversité biologique par le Secrétariat du CDB a révélé qu'aucun des objectifs d'Aichi pour la biodiversité n'a été atteint par les gouvernements et que seuls six d'entre eux ont été partiellement atteints au cours de la décennie écoulée ${ }^{4}$. La crise économique mondiale et les solutions de status quo ont mis en péril les objectifs environnementaux de la prochaine décennie avant même qu'ils ne soient officiellement ratifiés. La poursuite du développement économique au détriment de la justice sociale et environnementale fragilise les modes de vie des PA et des $C L$, ainsi que le bien-être et la santé de tous sur cette Planète. Pour sortir de cette pandémie avec des sociétés plus équitables et durables, il nous faut un changement inclusif et transformateur qui répare les injustices fondamentales telles que l'insécurité de la tenure, la marginalisation politique et la discrimination sociale des communautés qui gèrent plus de la moitié des terres.

Le présent document traite des évolutions législatives survenues pendant la crise de la COVID-19 en Inde, en Indonésie et aux Philippines qui compromettent les interactions durables entre l'homme et son environnement et, pour les PA et les $\mathrm{CL}$, la jouissance des droits sur leurs territoires coutumiers. Même si I'Inde, I'Indonésie et les Philippines n'ont toujours pas ratifié la Convention 169 relative aux peuples autochtones et tribaux, 1989 (OIT 169), ces trois pays ont déjà ratifié la Déclaration des Nations unies sur les droits des Peuples Autochtones (UNDRIP). Chacun de ces pays a également initié des réformes de tenure foncière et forestière au niveau national, même si leur mise en œuvre suit un rythme faible 5 .

Dans le présent document, nous présentons d'abord le contexte actuel, marqué par la crise de la COVID-19, dans chacun des pays cibles. Les processus législatifs présentés ici se déroulent parallèlement à la réponse de chaque pays face à la pandémie, y compris les mesures de confinement prises par l'État, ce qui donne un contexte important. Ensuite, nous résumons les évolutions législatives à travers trois thèmes, qui parfois se chevauchent :

1. Les avancées opportunistes dans les processus législatifs controversés précédant la COVID ;

2. La relance et la compensation des entreprises ;

3. Les solutions descendantes face à la pandémie fragilisant les droits des PA et des CL.

\footnotetext{
${ }^{3}$ Asia Indigenous Peoples Pact. (2020). Contribution de l'Asia Indigenous Peoples Pact (AIPP) et de ses organisations et réseaux membres en réponse partielle au «Rapport sur l'appel à contributions » du rapporteur spécial sur les droits des peuples autochtones auprès de l'Assemblée générale traitant de l'impact de la COVID-19 sur les Populations Autochtones en Asie. Pour le consulter, cliquez sur le lien suivant: https://aippnet.org/wp-content/uploads/2020/06/Final AIPP UNSRIP-June2020.pdf

${ }^{4}$ Secrétariat de la Convention sur la diversité biologique. (2020). Perspectives mondiales de la diversité biologique 5. Montréal. Pour le consulter : https://www.cbd.int/gbo/gbo5/publication/gbo-5-en.pdf

${ }^{5}$ Initiative des Droits et Ressources. (2018). At a Crossroads: Consequential Trends in Recognition of Community-Based Forest Tenure from 2002-2017 (À la croisée des chemins : Tendances dominantes dans la reconnaissance de la tenure forestière fondée sur la communauté de 2002 à 2017). Document de l'Initiative des Droits et Ressources, Washington D.C.:

https://rightsandresources.org/wp-content/uploads/2019/03/At-A-Crossroads RRI Nov-2018.pdf; Kumar, K., Singh, N. M., \& Rao, Y. G. (2017). Promise and Performance of the Forest Rights Act (Promesses et application de la loi sur les droits forestiers). Economic \& Political Weekly, 52(25-26).
} 
Nous avons constaté qu'en Inde, aux Philippines et en Indonésie, certaines initiatives gouvernementales poursuivent des programmes de développement antérieurs à la COVID et qui ont accru la probabilité de la prolifération et de la transmission, aux êtres humains, de maladies à transmission vectorielle telles que le COVID-196. Ces activités ont entravé le développement des programmes mondiaux de lutte contre le changement climatique et l'effondrement de la biodiversité, et continueront à le faire si les réponses à la pandémie centrées sur l'homme sont abandonnées au profit de solutions destructrices pour l'environnement.

\section{Droits humains pendant la COVID-19 : fermeture des espaces démocratiques}

Si le confinement est une importante composante stratégique dans les réponses visant à endiguer la propagation des agents pathogènes virulents, il est également utilisé par les autorités comme moyen d'empêcher toutes velléités de mobilisation des populations.

Les Philippines sont un cas extrême où des mesures de confinement et un état d'urgence national ont été utilisés pour accroitre la répression dans le cadre d'une réponse très militarisée à la COVID19. Le 24 mars 2020, le Président Duterte a consolidé ses pouvoirs exécutifs grâce à la loi de la République n 11469 (loi Bayanihan) qui délègue le pouvoir législatif au président en cas d'urgence nationale. Le Président Duterte peut désormais utiliser des fonds provenant d'autres branches de l'Etat afin de financer des plans de relance et des projets de développement. La loi de la République $n^{\circ} 11332$ subséquente a dégagé des orientations pour la quarantaine communautaire renforcée (QCR) en cours. Ensemble, ces deux lois sont utilisées en faveur du programme de développement de Duterte, mais elles constituent des leviers aux mains du pouvoir pour inhiber toute possibilité d'organisation de la population, et enfreignent davantage les droits civils, politiques et socioéconomiques des citoyens.

Le $1^{\text {er }}$ avril, le Président Duterte a prorogé une mesure permettant aux forces de sécurité de "tirer pour tuer", afin de pouvoir s'en prendre aux "fauteurs de troubles" qui protestent contre la QCR. Au cours des vingt premiers jours de la QCR, plus de 17000 personnes ont été arrêtées pour avoir enfreint les mesures de couvre-feu et de confinement. La QCR a eu d'importantes répercussions sur les PA et les $C L$ en ce sens qu'elle les a empêchés d'accéder aux ressources naturelles essentielles, aux activités économiques, à leurs champs et aux autres communautés ayant besoin d'aide. Par exemple, le 6 avril, une centaine de policiers ont violemment dispersé une barricade des Tuwali-Ifugao érigée pour arrêter la poursuite de l'exploitation minière d'OceanaGold. Plusieurs dirigeants autochtones ont été accusés d'avoir violé la QCR. La loi Bayanihan a expiré le 5 juin, et le 11 septembre, Bayanihan 2 a été promulguée par le Président Duterte, étendant ainsi ses pouvoirs spéciaux jusqu'au 19 décembre. Ainsi, un fonds supplémentaire de 34 millions de dollars a été créé pour faire face à la crise sanitaire, avec comme axe prioritaire la relance de l'économie.

Des exemples de criminalisation et de déplacement ont également été notés en Indonésie et en Inde. Pendant la pandémie, 37 nouveaux conflits agraires ont été enregistrés, 39 agriculteurs, autochtones et pêcheurs ont été arrêtés et deux personnes sont mortes dans des conflits agraires (données KPA).

\footnotetext{
${ }^{6}$ Les maladies zoonotiques sont courantes et leur prolifération est depuis longtemps liée à l'agriculture industrielle, aux marchés d'animaux sauvages, à la déforestation et à la dégradation de l'environnement. Ces dynamiques créent l'environnement idéal pour que les agents pathogènes puissent rapidement proliférer et muter à travers des réservoirs d'espèces qui, autrement, n'entreraient pas en contact étroit avec des populations humaines plus importantes. La COVID-19, le changement climatique, la perte de biodiversité et les activités économiques qui contribuent à la dégradation de l'environnement sont tous liés.
} 
L'accaparement des terres des agriculteurs, les expulsions forcées et les réinstallations de communautés hors de leurs zones d'habitation se poursuivent, alors même que l'Indonésie est confrontée à une crise sanitaire et alimentaire ainsi qu'à une récession économique causée par la pandémie.

En Inde, la mesure de confinement prise en mars a bloqué des millions de travailleurs migrants dans les zones urbaines, ce qui les a obligés à marcher des centaines de kilomètres pour rentrer chez eux, tout en faisant face à la faim, à la répression policière et à la mort. Cependant, les restrictions de déplacement n'ont pas été appliquées au secteur minier, qui est jugé essentiel, et les pratiques de reboisement compensatoires ont poursuivi la déforestation puis la reforestation de terres revendiquées et utilisées par les PA et les CL. ${ }^{7}$ Au cours des derniers mois, il y a eu une série de déplacements, notamment pour un réservoir et des initiatives de conservation. ${ }^{8}$ Entre-temps, l'intimidation envers les activistes s'est intensifiée. Par exemple, la section indienne de Fridays for Future, un mouvement mondial de grève pour le climat mené par des jeunes, a reçu un préavis le $\underline{8}$ juillet en vertu de la loi (de prévention) des activités illégales (UAPA) et son site internet a ensuite été bloqué. Suite à l'indignation générale suscitée par la qualification de "terroristes" attribuée à de jeunes écologistes, la police de Delhi a retiré son préavis, prétextant ce qu'elle a qualifié d'erreur administrative. L'UAPA est une loi relative au terrorisme et a été utilisée contre Fridays for Future en raison de sa campagne contre le nouveau projet EIE 2020 (voir la première partie ci-dessous). Dans son préavis, la police de Delhi reprochait à Fridays for Future de perturber "la paix, [et] la souveraineté de l'Inde".

\section{1ère Partie: Processus législatifs controversés}

Avant la crise de la COVID-19, les trois pays poursuivaient des programmes de développement néolibéraux qui favorisaient les intérêts des élites transnationales et locales, fragilisant ainsi l'intégrité environnementale des paysages communautaires. Nombre de ces lois ont fait l'objet d'une résistance acharnée dans les mois qui ont précédé la COVID-19. C'est le moratoire sur les rassemblements publics qui a été utilisé pour mettre fin à la résistance.

En Indonésie, le gouvernement du Président Joko Widodo utilise la COVID-19 pour promouvoir des processus législatifs controversés largement critiqués avant la pandémie. De larges pans de la société civile considèrent que ces processus affaiblissent les mesures de protection environnementale existantes, et permettent aux entreprises de se constituer en banques foncières et de porter atteinte à l'intégrité des communautés autochtones Adat d'Indonésie et des communautés locales qui gèrent les ressources de façon traditionnelle et durable.

- La loi Omnibus pour faciliter l'octroi de licences aux entreprises. La loi sur la création des lieux de travail, connue sous le nom de loi Omnibus, a été votée le 5 octobre malgré la forte opposition des organisations paysannes, environnementales et de PA, des syndicats et du mouvement estudiantin. II s'agit d'un ensemble de réformes de grande envergure qui déréglementent les entreprises à travers plus de 1000 amendements contenus dans quelque 79 lois. La loi Omnibus déréglemente l'exploitation minière, réduit les sanctions pour violation de l'environnement, supprime les exigences en matière d'EIE, crée des conditions favorisant la corruption et exclut complètement les PA et les $\mathrm{CL}$ des processus de prise de décisions. Les

\footnotetext{
${ }^{7}$ La pratique consistant à considérer l'exploitation minière comme un service « essentiel » a été observée dans d'autres pays tels que l'Afrique du Sud, le Pérou, le Canada, la Guyane, etc.

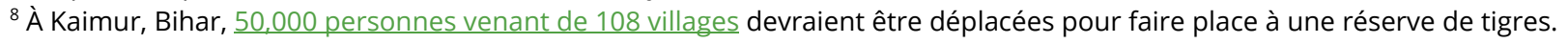


amendements à la loi Omnibus se heurtent aux lois sur la décentralisation en Indonésie et sont susceptibles de déclencher des batailles judiciaires. Depuis la ratification du projet de loi, plusieurs versions ont circulé, chacune avec des différences importantes, ce qui entraîne un manque de clarté sur la forme finale que prendra la loi Omnibus. Une différence importante étend l'interdiction de pratiques traditionnelles de subsistance utilisant le feu pour défricher les terres agricoles, tout en rendant difficile de sévir contre les plantations qui utilisent le feu pour défricher les forêts. II est également apparu que douze hommes politiques ayant contribué à la promotion de la loi ont des liens avec le secteur minier. Au cours des deux semaines suivant le début des manifestations, plus de 6000 personnes ont été arrêtées. La vague de protestations que suscite cette nouvelle loi est menacée par la loi sanitaire sur la quarantaine, qui n'a pas été utilisée jusqu'à présent.

- L'accaparement de terres via des banques foncières. La loi Omnibus est accompagnée du décret présidentiel (Perpres) $n^{\circ} 662020$ sur les marchés publics fonciers pour les projets de développement d'intérêt public. Perpres $n^{\circ} 66$ a été signé et promulgué le 19 mai 2020 et a réintroduit les dispositions d'un projet de loi foncière controversé en ce qui concerne le concept de banque foncière. Cela va considérablement accélérer l'accaparement des terres dans les territoires des PA/CL et mettre en danger de vastes étendues de paysages forestiers intacts et des territoires habités par des PA et des CL.

- La reconnaissance des forêts coutumières est lente. Le Ministère de l'Environnement et des Forêts a publié la nouvelle réglementation nº 17 de 2020 relative aux forêts coutumières, rallongeant les procédures de reconnaissance et de protection des forêts coutumières. Les demandes de reconnaissance des forêts coutumières en attente soumises par les PA, ainsi que les cartes de leurs territoires, représentent déjà 6 millions d'hectares. La publication de cette nouvelle réglementation ministérielle pourrait compliquer davantage la reconnaissance et la protection des forêts coutumières.

Le gouvernement BJP de I'Inde tente systématiquement de faire reculer les protections relatives aux droits de l'homme et à l'environnement sous le prétexte d'encourager les investissements pendant la pandémie. Le secteur minier a été au centre de ces reculs.

- Le projet d'évaluation d'impact environnemental (EIE) 2020. Le Ministère de l'Environnement, des Forêts et du Changement Climatique (MoEFCC) a publié un projet de notification EIE 2020 en mars 2020 qui propose de réduire le nombre d'audiences publiques, affaiblit le processus de consultation, amnistie les projets coupables d'infractions environnementales, et dispense certaines catégories de l'industrie de la nécessité de procéder à des EIE. Au total, douze des quatorze clauses de la précédente EIE 2006 ont été amendées dans le nouveau texte. Les mines désirant étendre leurs activités ont également été dispensées de l'obligation d'obtenir une autorisation environnementale (AE). Cela est en totale contradiction avec le fondement statutaire de la loi sur (la protection de) l'environnement de 1986, entravant les objectifs de l'Inde en matière de biodiversité et climat. Le projet d'EIE 2020 aura un impact particulier sur la région nord-est du pays, où vivent d'innombrables tribus protégées par le sixième avenant de la Constitution indienne. L'article 371 et le sixième avenant accordent aux communautés la propriété de leurs ressources naturelles, mais la disposition du projet d'EIE 2020 visant à créer des industries à moins de $100 \mathrm{~km}$ des frontières internationales sans consultation publique aura un impact disproportionné sur ces communautés. La fragilisation des dispositions relatives à l'EIE dans le projet EIE 2020 aura probablement de graves répercussions à long terme, de par la limitation de la supervision et la non-prise en compte des risques socio-écologiques. En outre, le projet hydroélectrique 
controversé de $3097 \mathrm{MW}$ d'Etalin ne tient pas compte de l'activité sismique, des risques glaciaires liés au changement climatique, des glissements de terrain et des conséquences sur les communautés qui découleraient d'une catastrophe naturelle. En fin de compte, le projet EIE 2020 encourage le développement technocratique au détriment des droits de l'homme et de l'environnement, des préoccupations qui ont également été soulevées par les rapporteurs spéciaux des Nations unies.

Aux Philippines, le régime Duterte est connu pour sa propension à museler l'opposition, pour ses exécutions extrajudiciaires et pour ses mesures répressives contre toute dissidence. ${ }^{9}$ Un rapport de Global Witness sur les défenseurs des droits fonciers et de l'environnement a révélé que 43 d'entre eux ont été tués, ce qui fait des Philippines le deuxième pays le plus meurtrier pour les défenseurs des droits en 2019 derrière la Colombie. ${ }^{10}$ Pris ensemble, ces développements soulèvent de graves inquiétudes pour l'avenir.

- La dissidence assimilée au terrorisme. Pendant la pandémie, le projet de loi du Sénat n 1083 a été approuvé tandis que la Chambre des représentants a approuvé le projet de loi $n^{\circ} 6875$. Ces deux lois ont été consolidées pour créer la loi de la République 11479, ou loi antiterroriste de 2020, que le Président Duterte a ensuite signée le 18 juillet 2020 pour remplacer la loi sur la sécurité humaine de 2007. Cela a été fait malgré les critiques de la communauté internationale, notamment celles du $\mathrm{HCDH}$. La loi antiterroriste brouille les frontières entre l'activisme juridique, la dissidence et le terrorisme, alimentant ainsi les craintes d'abus de pouvoir continus et accrus de la part du gouvernement Duterte. ${ }^{11}$

- Criminalisation des Peuples Autochtones. Une loi controversée passée dans les mois précédant la pandémie et qui a fait l'objet d'une forte résistance, le décret $n^{\circ} 70$ adopté en décembre 2018, ${ }^{12}$ est largement utilisée pendant la pandémie pour faire taire les dissidents et criminaliser davantage les défenseurs des droits des Peuples Autochtones (IPHRD). En ciblant les organisations et en militarisant davantage les territoires des Peuples Autochtones, le décret $\mathrm{n}^{\circ} 70$ amplifie le ciblage sur liste rouge et les menaces envers les militants.

\section{2ème Partie: Relance et compensation des entreprises}

Les petites et moyennes entreprises dépendent souvent du soutien de l'Etat pour survivre aux crises. Cependant, en réponse à la COVID-19, les gouvernements donnent la priorité à l'expansion du secteur de l'industrie extractive et aux projets de développement à grande échelle par des dispositions qui régiront l'environnement économique pour de nombreuses années à venir. Sous le prétexte de la relance économique nécessaire face à la COVID-19, les États ont mis en ceuvre un certain nombre de stratégies favorables aux entreprises. II s'agit, entre autres, de la déréglementation, la rationalisation des licences ou l'octroi d'avantages fiscaux. Les territoires des PA et des CL sont ciblés pour des activités économiques qui contribueront à la dégradation de l'environnement et au changement climatique.

\footnotetext{
${ }^{9} \mathrm{~A} / \mathrm{HRC} / 44 / 22$

${ }^{10}$ Les Philippines étaient le pays le plus meurtrier pour les défenseurs des droits en 2018.

${ }^{11}$ Les PA et les CL sont déjà blacklistés et étiquetés comme sympathisants communistes, emprisonnés pour avoir défendu leurs maisons ancestrales contre les projets d'extraction et d'infrastructure et empêchés de s'organiser. La loi antiterroriste de 2020 sera probablement utilisée pour réprimer la dissidence et toute critique du gouvernement, y compris celles venant des PA, des $\mathrm{CL}$ et de leurs organisations.

${ }^{12}$ Le titre complet du décret $n^{\circ} 70$ est « Institutionnaliser l'approche globale nationale pour parvenir à une paix inclusive et durable, créer un groupe de travail national pour mettre fin au conflit armé communiste local et mener l'adoption d'un cadre national pour la paix ».
} 
L'Indonésie a indemnisé les entreprises par le renouvellement automatique des licences et la levée des exigences certifiant la légalité des produits. Dans certains cas, la mobilisation du public a permis de contrer ces reculs, mais plusieurs dispositions compensatrices qui ont été adoptées auront des conséquences à long terme sur les forêts indonésiennes et pour les PA et CL qui y vivent.

- Prolongation automatique des concessions minières de charbon. La loi sur les minerais et le charbon (loi Minerba) a été révisée sans aucune participation de la population. L'article 47 garantit aux compagnies minières une double prolongation de 10 ans. Dès lors, la nouvelle loi garantira la prolongation automatique des concessions minières, assurant ainsi la domination de l'oligarchie de l'industrie houillère pendant encore 20 ans.

- Renouvellement automatique des licences pour les plantations, le bois et les concessions de pâte à papier. Le Ministère de l'Agriculture indonésien a annoncé un renouvellement automatique des licences jusqu'en fin 2020 pour compenser les pertes des entreprises du fait de la COVID.

- Coup de fouet à l'industrie illégale du bois. Le Ministère du Commerce a tenté de révoquer l'exigence de légalité pour les exportations de bois. L'Indonésie a initié un système de vérification de la légalité du bois (SVLK) depuis 2003, et la suppression de l'exigence de légalité aurait des conséquences majeures sur la déforestation et la surveillance du bois d'origine illégale. Depuis que cette mesure a pris effet le 27 mai 2020, les craintes concernant l'inondation du marché par le bois illégal, ${ }^{13}$ la déforestation et la sécurité territoriale s'accentuent. Cependant, les organisations de la société civile (OSC), les universitaires, le secteur privé et même le Ministère de l'Environnement et des Forêts (MEF) se sont mobilisés avec succès pour que l'exigence de légalité soit rétablie.

Plutôt que de renforcer ses dispositions environnementales mal appliquées, I'Inde revient sur les protocoles qui définissent et préviennent les violations. Ces évolutions s'accompagnent de l'incapacité à inclure et à consulter de manière adéquate les PA et les $C L$ dans les projets ayant un impact direct et indirect sur leurs modes de vie traditionnels.

- Les lois indiennes sur l'environnement ne prévoient pas de dispositions relatives aux autorisations par vidéo. L'approbation rapide de l'agrément environnemental (AE) pour les projets risque d'entraîner des cas d'oubli d'ordre administratif ${ }^{14}$. Entre le 24 mars et le 30 juin, une analyse des procès-verbaux des réunions du comité consultatif sur les forêts et du comité d'expertise de l'évaluation a montré qu'environ 120 projets ont été recommandés, 90 reportés, 30 retournés, et deux rejetés. Les réunions se sont déroulées par vidéoconférence en raison du confinement. Un quart des projets recommandés se situaient dans les districts du cinquième avenant et ont été approuvés sans la diligence requise ou le respect des droits

\footnotetext{
${ }^{13} 40 \%$ des produits issus du bois importés en Europe vient d'Indonésie.

${ }^{14}$ Le 27 mai, une explosion et un incendie se sont produits dans un puits de pétrole appartenant à Oil India Ltd (OIL) à Assam, 17 jours après que le projet Baghjan OIL a reçu I'AE du MEFCC. OIL a trois autres projets d'exploration pétrolière et gazière qui attendent l'AE du MEFCC. Étant donné que tous les projets d'exploration pétrolière et gazière sur terre et en mer relèvent de la catégorie "B2" et sont exemptés d'audiences publiques, les propositions de I'OIL ne feront pas l'objet d'une procédure d'audience publique. Compte tenu des inondations annuelles le long Brahmapoutre, et de la proximité de l'explosion avec le fleuve et des écosystèmes vulnérables, l'incident pose de graves risques pour les PA et les CL ainsi que pour la faune sauvage en Inde et en aval au Bangladesh. Fin juin 2020, les efforts pour contenir les incendies ont été entravés par ces mêmes inondations. Cela montre à quel point les dispositions déjà faibles qui ont facilité des projets tels que Baghjan OIL doivent être renforcées et non pas affaiblies ou supprimées complètement. Le projet Baghjan OIL a été approuvé dans le cadre de l'EIE 2006, accentuant ainsi la nécessité de renforcer les dispositions de l'EIE plutôt que de les affaiblir comme le projet EIE 2020.
} 
inscrits dans la loi sur les droits forestiers et au mépris du droit au CLIP. Au total, 30 projets approuvés par le MoEFCC se trouvent dans des zones de biodiversité vulnérables

- Commercialisation du secteur de l'extraction du charbon. Le 11 juin 2020, le gouvernement indien a lancé une nouvelle vente aux enchères pour l'extraction de charbon, sur le thème "Libérer le charbon : de nouveaux espoirs pour Atmanirbhar Bharat", impliquant 41 mines de charbon dans cinq États. Cette mesure a été accompagnée d'autres lois, comme l'amendement de la section 8A de la loi de 1956 sur les mines et les minerais (réglementation et développement), qui prorogent automatiquement toutes les approbations, licences et droits valides en faveur du soumissionnaire retenu pour les baux miniers. Ces approbations ont en outre été dispensées des autorisations nécessaires imposées par la loi sur la conservation des forêts de 1980 et la loi sur les droits forestiers de 2006 pour une période de deux ans. Un amendement de la loi de 2015 sur les mines de charbon (dispositions spéciales) permet, pour la première fois à des entreprises privées, d'exercer des activités dans l'industrie du charbon. Ces réformes de grande envergure dans le secteur minier indien, en particulier le charbon, constituent une mesure sans précédent qui compliquera davantage la mise en œuvre des réformes de tenure forestière introduites par la loi de 2006 sur les droits forestiers 2006 (loi sur les droits forestiers). Ces mines de charbon sont également situées sur des terres autochtones et des forêts d'une grande diversité biologique.

- Suppression des mesures de protection du travail. Dans leur effort d'accompagnement des entreprises, les États indiens et le gouvernement central sont en train de démanteler systématiquement les droits des travailleurs afin d'encourager la croissance économique. Ces réformes d'envergure vont affaiblir les mesures de protection en termes de temps de travail, de salaires, d'heures supplémentaires, de santé, de sécurité au travail etc. En faisant cela, I'Inde fera peser le fardeau financier de la relance économique sur ses populations les plus vulnérables, qui sont souvent les groupes les plus durement affectés par le confinement imposé par le gouvernement.

- Ouverture des terres agricoles à l'industrie. L'État d'Assam a adopté une ordonnance sur les micro, petites et moyennes entreprises (MPME) visant à faciliter les affaires, en supprimant la nécessité d'obtenir une autorisation, une habilitation ou des licences pour ouvrir des terres agricoles à des activités industrielles. Le gouvernement central indien envisage également de créer une société nationale de gestion des terres afin de faciliter la monétisation des terres "inutilisables" sur le papier mais qui, dans la pratique, sont gérées de façon coutumière par les communautés. Cela entraînera des conflits fonciers, des déplacements, la perturbation des moyens de subsistance et la criminalisation des populations vulnérables qui ne jouissent pas d'un régime de tenure garanti sur leurs terres et leurs ressources. Le gouverneur du Karnataka a promulgué l'ordonnance (amendement) sur les réformes foncières du Karnataka, 2020, qui modifiera la loi sur les réformes foncières du Karnataka, $1961^{15}$. Cela permet l'ouverture des terres agricoles aux non-agriculteurs et modifie les plafonds fonciers. Les associations d'agriculteurs protestent contre cette mesure qui ouvre la voie à des acquisitions de terres à grande échelle qui favoriseront des acteurs non étatiques plus importants et plus puissants.

Suite à la QCR et au renouvellement des pouvoirs exécutifs, les Philippines ont vu des fonds venant d'autres branches du gouvernement être utilisés en faveur de plans d'infrastructure ciblant les terres

\footnotetext{
${ }^{15}$ L'amendement promulgué le 13 juillet 2020 supprime les articles 79A, 79B et 79C de la loi qui imposent des restrictions strictes à la propriété des terres agricoles.
} 
ancestrales. Ces secteurs peuvent poursuivre leurs activités, ce qui facilitera potentiellement la propagation de la COVID-19 dans les zones rurales éloignées.

- L'infrastructure, solution de fortune face à la pandémie. Le 4 juin 2020, le gouvernement a adopté le projet de loi $n^{\circ} 6815$ de la Chambre des représentants, intitulé "Accélération de la reprise et relance des investissements pour l'économie des Philippines" (ARISE Philippines), un plan de relance de 2,6 milliards de dollars. Environ $50 \%$ des fonds ont été alloués à des projets infrastructurels destinés à relancer l'économie. Seulement, le budget a depuis lors été réduit à 340 millions de dollars, ce qui fait de l'enveloppe allouée à la relance budgétaire des Philippines l'une des plus faibles au monde. Parmi les projets d'infrastructures qui seront soutenus par le plan de relance, plusieurs se trouvent sur les terres ancestrales des PA et des $\underline{C L}$ et font l'objet de beaucoup de contestations ${ }^{16}$. Le projet n'est pas encore devenu une loi.

- Un projet de développement axé sur le marché cible les terres ancestrales. Le programme-phare "Build Build Build" (BBB, construire, construire, construire) du plan de développement des Philippines (PDP) 2017-2022 a été redynamisé suite à la pandémie, avec comme objectif de promouvoir "l'Age d'or" des infrastructures. Il y a plus de 100 projets au titre du BBB, dont beaucoup ciblent les territoires ancestraux des PA et des CL. Plus de 110000 personnes autochtones venant d'au moins 106 villages pourraient être touchées par les cinq projets de barrages proposés, sans compter les 230 autres demandes d'exploitation minière approuvées empiétant sur au moins 542.245 hectares de terres ancestrales. ${ }^{17}$ Le Président Duterte utilise ses pouvoirs exécutifs aux termes de la Loi Bayanihan (étendue dans Bayanihan 2) pour réaffecter des fonds tirés d'autres secteurs du gouvernement, grâce à la loi du Sénat $n^{\circ} 1474$, priorité accordée aux prêts en faveur des projets d'infrastructure de BBB, avec un montant d'1,3 milliard de dollars dégagé pour l'exercice 2021

3ème Partie: Face à la pandémie, des "solutions" qui sapent les droits des PA / CL

Une pandémie prolongée augmente les risques de crise alimentaire. Les mesures de confinement ont eu un impact considérable sur la capacité des communautés à se nourrir, à accéder aux équipements de base et aux services de santé. Sapant les droits des PA et des CL, ces "solutions" mettent à rude épreuve ces groupes, qui peinent à collecter, récolter, cultiver et distribuer les denrées alimentaires.

- Accès limité aux forêts. Dans une note publiée le 6 avril 2020, le MoEFCC a demandé à tous les États et territoires de limiter les interactions entre I'homme et la faune en réduisant la circulation des personnes dans les parcs nationaux, les sanctuaires et les réserves de tigres. Les PA et les CL dépendent fortement des produits forestiers non ligneux (PFNL) pour leur subsistance et ces restrictions auraient un impact immédiat pour 3 à 4 millions de personnes vivant à l'intérieur et autour des zones protégées. En limitant l'accès des communautés rurales aux ressources forestières qui leur fournissent nourriture et médicaments, on touche à un aspect essentiel à leur survie. Les mesures de confinement ont également coïncidé avec la saison des récoltes, compromettant ainsi la survie de quelque 275 millions de personnes dans tout le pays.

- Des plantations de riz sur des terres contestées. Le président indonésien Joko Widodo a demandé aux entreprises publiques d'ouvrir de nouvelles rizières sur 900000 hectares dans

\footnotetext{
${ }^{16}$ Voir note antérieure. Informations fournies par Cordillera Peoples Alliance.

17 Informations fournies par Cordillera Peoples Alliance.
} 
le centre de Kalimantan. De nombreuses zones proposées sont des terres agricoles traditionnelles des PA et des $\mathrm{CL}$ ainsi que d'importantes tourbières. Ce programme gouvernemental proposé est susceptible de provoquer davantage de conflits agraires et d'accaparement de terres, et risque d'entraîner de graves conséquences pour la contribution indonésienne à l'Accord de Paris s'il n'est pas fait pour et par la population.

- Ciblage des terres des PA/CL pour la production alimentaire. Le Direction de l'Agriculture des Philippines (DA) et la Commission nationale des Peuples Autochtones (NCIP) ont publié une proposition conjointe pour augmenter la production alimentaire qui cible les terres ancestrales dites "inoccupées" des PA et des CL. II est reproché au programme "Plant Plant Plant" (PPP, planter, planter, planter) d'ouvrir les terres ancestrales à l'agriculture industrielle, permettant ainsi l'accaparement des terres. Ces terres ne sont pas inoccupées, et le PPP va entraver l'action collective et les pratiques coutumières des PA et des CL qui militent pour une utilisation durable des ressources naturelles.

\section{Conclusion}

La pandémie de la COVID-19 a aggravé les vulnérabilités des PA et des CL. La criminalisation, la corruption, les menaces, les violences sexistes, les industries extractives, l'inaction de l'État et l'accès restreint à des informations culturellement appropriées ont tous empiré. Pourtant, ces communautés restent résilientes et parviennent à faire face collectivement à la pandémie avec succès. Cette réussite repose sur des actions autodéterminées, des systèmes de gouvernance traditionnels et des droits de tenure collectifs garantis sur les terres et les ressources.

La solidarité entre les organisations représentant les PA et les CL a été la pierre angulaire de cette réponse. Par exemple, dans toute l'Indonésie, les communautés ont commencé à se préparer à une crise alimentaire dès le mois de mars selon les principes de Wilayah Bermartabat (un territoire digne). La solidarité entre zones rurales et urbaines est mise en œuvre par le Mouvement de solidarité des greniers agricoles du Consortium pour la réforme agraire (KPA), à travers lequel les agriculteurs et les pêcheurs excédentaires donnent ou vendent leurs produits à bas prix à destination des travailleurs urbains. Cet exemple souligne également l'importance de la tenure foncière communautaire: la majorité des produits soutenus par le KPA ont été cultivés sur des terres contestées.

Les communautés ont également mis à profit leurs droits fonciers pour répondre à la crise. Des villages en Inde tels que Kukdale dans le District de Gadchiroli au Maharashtra, ont tiré parti de leur indépendance économique qui repose sur des droits communautaires sur les ressources forestières, pour venir en aide aux familles les plus vulnérables en leur fournissant des vivres à fréquence hebdomadaire. À Riau, une province de l'île de Sumatra en Indonésie, les jeunes Talang Mamak contribuent à la sécurité alimentaire de leurs villages et encouragent le contrôle communautaire. Ces deux exemples sont multiples et existent dans des centaines de milliers de villages de PA et CL qui se sont organisés pour assurer la santé, la sécurité et les moyens de subsistance des membres de leur communauté.

Il est essentiel que les législateurs et les dirigeants du monde post-COVID s'inspirent de ces actions dans leurs réponses économiques. Les droits et l'autodétermination des PA et des CL doivent figurer en bonne place dans les programmes de relance pour sauvegarder la nature et prévenir les risques de futures pandémies. Aucune relance économique ne peut prospérer si l'on compromet l'espace démocratique des communautés les plus aptes à protéger les ressources de la planète. 
-13 - 


\section{À propos de l'Initiative des Droits et Ressources (RRI)}

L'Initiative des Droits et Ressources est une Coalition mondiale de plus de 150 organisations engagées à faire progresser les droits relatifs aux forêts, aux terres et aux ressources des Peuples Autochtones, des Afro-descendants, des Communautés Locales et des femmes au sein de ces groupes. Les membres tirent parti des forces, de l'expertise et de la portée géographique de chacun pour trouver des solutions plus efficaces. RRI s'appuie sur l'expertise des membres de la Coalition pour faire l'écho de la voix des peuples locaux et pousser les gouvernements, les institutions multilatérales et les acteurs du secteur privé à adopter les réformes institutionnelles et commerciales en faveur de la réalisation de ces droits. En développant une compréhension stratégique des menaces et opportunités mondiales résultant de droits précaires sur les terres et les ressources, RRI élabore et promeut des approches commerciales et de développement fondées sur les droits et catalyse des solutions efficaces pour renforcer la réforme du régime foncier rural et la gouvernance durable des ressources.

RRI est coordonné par le Groupe pour les Droits et les Ressources (RRG), une organisation à but nonlucratif basée à Washington, DC. Pour plus d'informations, visitez le site : https://rightsandresources.org/fr/.

\section{Partenaires}

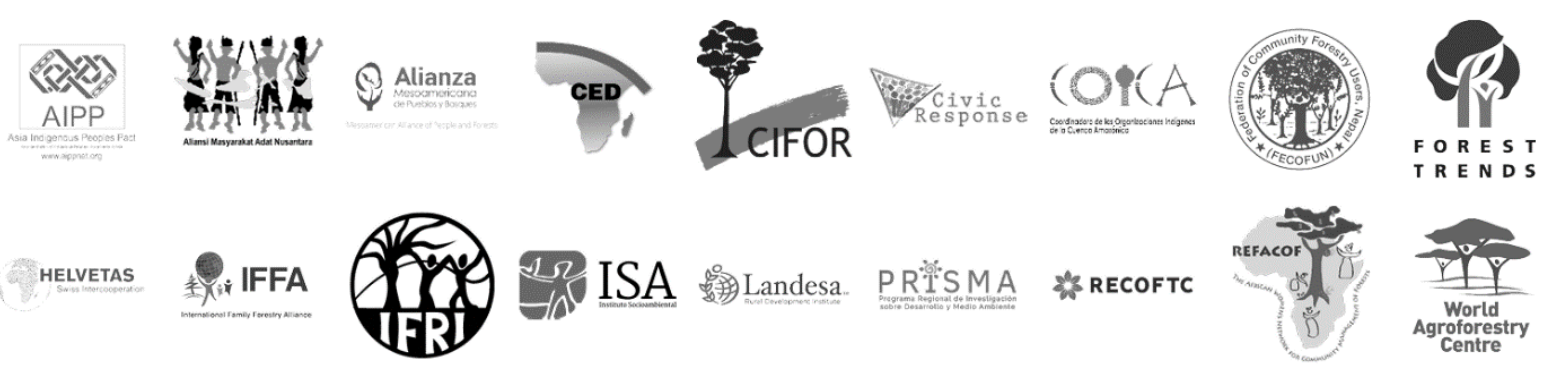

Sponsors
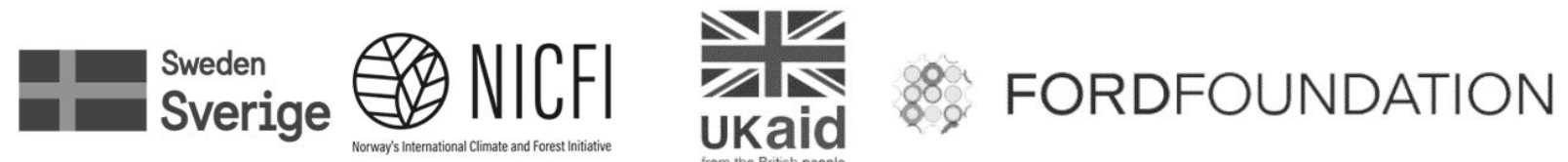

Les opinions présentées ici ne sont pas nécessairement partagées par les organismes qui ont généreusement soutenu ce travail. Ce travail est sous licence Creative Commons BY 4.0. 